\title{
The Second American Cough Conference
}

\author{
Peter V. Dicpinigaitis
}

Published online: 28 July 2009

(C) Springer Science+Business Media, LLC 2009

Two years after our inaugural meeting [1], the Second American Cough Conference was held in June 2009 on the banks of the Hudson River, in the shadow of the New York City skyline. Once again, a faculty of internationally renowned basic and clinical scientists provided state-ofthe-art summaries and updated developments in the recently energized field of cough. The audience filling the auditorium to capacity comprised a broad range of interested parties: researchers, academicians, clinicians, and members of the pharmaceutical industry.

Reflecting the multidisciplinary challenge that pathological cough presents, conference speakers represented a broad range of backgrounds: basic and preclinical science, respiratory medicine, allergology, otorhinolaryngology, biomedical engineering, and pediatrics. As is the goal of the American Cough Conference, new and emerging themes, involving mechanisms, diagnosis, and management of cough, were presented and discussed. Notable among these is the concept that a preexisting, heightened cough reflex may underlie the development of chronic cough in susceptible individuals upon the superimposition of a second, exacerbating factor such as acute viral upper respiratory tract infection or gastroesophageal reflux, whereas in others with less sensitive baseline cough reflex sensitivity, identical triggers will not result in the onset of cough [2]. New developments in the pharmacology of cough were prominently featured, including an update on the TRP family of ion channels. Whereas the TRPV1 ion channel has received significant attention as a potential target for antitussive drugs

P. V. Dicpinigaitis $(\square)$

Albert Einstein College of Medicine and Montefiore Medical

Center, 1825 Eastchester Road, Bronx, NY 10461, USA

e-mail: pdicpinigaitis@pol.net
[3], the TRPA1 channel is now identified as a particularly exciting area of investigation. Recently emerging areas of inquiry, including the association of cough with sleep, exercise, and laryngeal sensory neuropathy, were discussed and new data presented. The urgent need for new and better antitussive drugs was a recurrent theme throughout the meeting, as was perhaps also the sentiment that the most efficient path to that goal would involve collaboration between academia and the pharmaceutical industry.

The Second American Cough Conference could not have occurred without the generosity of our major financial sponsor, Reckitt Benckiser, as well as our other providers of significant support, Procter \& Gamble and Wyeth Pharmaceuticals. We are grateful to F. Dennis McCool, Editor-in-Chief of Lung, and the publisher, Springer, for again publishing the proceedings of our conference in their journal. We thank the SRxA Institute for Professional Education for cosponsoring the conference along with Medical Education Resources. Finally, we are greatly indebted to the administrative organizers of our meetingChris Efessiou, Ph.D., Jenny Mauk, M.Ed., and Veronica Pecnik of Strategic Pharmaceutical Advisors and the SRxA Institute for Professional Education-for their meticulous oversight of every aspect and detail of our conference.

The Third American Cough Conference is planned for June 2011. Updates regarding this meeting will be provided, as they become available, on our website: www.cough conference.com.

\section{References}

1. Dicpinigaitis PV (2008) The First American Cough Conference. Lung 186(Suppl 1):S1-S2 
2. McGarvey L, McKeagney P, Polley L, MacMahon J, Costello RW (2009) Are there clinical features of a sensitized cough reflex? Pulm Pharmacol Ther 22:59-64
3. McLeod RL, Correll CC, Jia Y, Anthes JC (2008) TRPV1 antagonists as potential antitussive agents. Lung 186(Suppl 1): S59-S65

\section{Financial Disclosure/Conflict of Interest Statement}

The authors of this article have no commercial associations (e.g., consultancies, stock ownership, equity interests, patent-licensing arrangements, etc.) that might pose a conflict of interest in connection with the submitted article, except as disclosed on a separate attachment. All funding sources supporting the work and all institutional or corporate affiliations of the authors are acknowledged in a footnote. 